\title{
Pen vs. Computer - how to write by hand Improves Memory and Creativity
}

\section{Dario Furnari \\ Magazines:stylus}

Corresponding Author: Dario Furnari, Magazines: stylus.

Received date: August 29, 2019; Accepted date: Septembe 10, 2019; Published date: September 17, 2019

Citation : Dario Furnari. (2019) PEN vs. COMPUTER - how to write by hand improves memory and creativity. J. Neuroscience and Neurological Surgery. 5(1); DOI:10.31579/2578-8868/188

Copyright : ( 2019 Dario Furnari. This is an open-access article distributed under the terms of The Creative Commons Attribution License, which permits unrestricted use, distribution, and reproduction in any medium, provided the original author and source are credited.

The pen is one of the many channels for transforming thoughts into written words. Handwriting acts as a "memory propeller". For example, when you are at the supermarket, the simple act of writing keeps the products you really need in your mind; or when someone at school was a shrewd knows that very often there was no need to use the sheets prepared before a class assignment to copy because they remembered everything that was written. This memory would have failed if the ticket to copy had been written or better typed on the keyboard. The latter characterizes the work of many people and yet, even today, children make their first experiences of writing with a pen or pencil. But is it really out of fashion? Today we have the opportunity to write our thoughts in other ways, "keyboard, tablet, touch screen", and even the signature, once the test par excellence of our identity, is faced with the competition of our " Fingerprints". Indeed, in some American schools the writing, replaced by the $\mathrm{PC}$, is no longer included in the didactic plans; according to some scientists and promoters of this event, this method would facilitate children's learning and improve creativity. So, what can we do? Fortunately, we come to the real "scientific research" based on real scientific protocols for the progress of science and not for some advertising promotion.

A team of researchers coordinated by the Psychologist Sandra Sulzenbruck of the Leibniz Institute in Dortmund hypothesizes that handwriting facilitates and stimulates "fine motor skills". The research consists of a comparison with some right-handed people who write a lot with the keyboard and other right-handed people who instead make much use of paper and pen. Both groups were asked to carry out a battery of skill tests, changing the use of the hand.

Research has shown that "technology" takes longer to do the exercises but only when they use the right; it is evident that these individuals are not used to a precise coordination of movements.

The most serious effect, however, concerns above all the cognitive sphere. According to the French neuro-scientist Marieke Longcam, both children and adults find it easier to learn new signs of writing, or a new language, when they reproduce them "by hand" instead of the PC.

During the experiment some participants practiced writing the letters by hand, the others with a special keyboard. After the trial, after several weeks, the researchers verified the successes of learning; result: those who had written freehand were much better, as they were able to distinguish with greater precision the graphic gestures, learned in the previous lesson, from other similar but reversed characters.

In our alphabet the reversed letters are the "b- $\mathrm{d}-\mathrm{p}-\mathrm{q}$ "; and it is precisely on these letters that children encounter their own difficulties especially if they are dyslexic. Also write these letters to one

keyboard does not change anything from the motor point of view; since every graphic sign, for example take the "s", has a particular movement of the body, different from the act of typing on a keyboard. From the neurophysiological point of view, using fMRI (functional magnetic resonance imaging) it is possible to visualize the graphs of brain activity.

Still in the experiment, each participant who had tried to write graphic signs by hand, had memorized certain movements associated with each graph-motor sign. When they tried to remember, the tomograph showed an increase in neuronal activity in the left Broca area, in the anterior intraparietal area and in the left premotor dorsal cortex; all areas dedicated to the mental development of an action. Thus, increased motor involvement implies better "synaptic plasticity" on learning, a more stable connection in memory and a better repetition of information.

Now, when was the last time you used the pen? Would Manzoni or Dante have written their works anyway if they had had a personal computer available?

Think people think.

\section{References}

1. Sulzenbruck S. (2011) The Death of Handwriting: Secondary Effects of Frequent Computer Use on Basic Motor Skills, in "Journal of Motor Behavior",

2. LongCamp M. (2008) Learning through Hand- or Typewriting influences Visual Recognition of New Graphic Shapes: Behavioral and Functional Imaging Evidence, in "Journal of Cognitive Neuroscience", 